\title{
ROLE OF THE RIGHT PREFRONTAL CORTEX IN ILL-STRUCTURED PLANNING
}

\author{
Vinod Goel \\ York University, Toronto, Canada \\ Jordan Grafman \\ National Institutes of Health, Bethesda, USA
}

\begin{abstract}
We tested an architect with a lesion to the right prefrontal cortex in a real-world architectural design/ planning task that required him to develop a new design for our lab space and compared his performance to an age- and education-matched architect.

The patient understood the task and even observed that "this is a very simple problem." His sophisticated architectural knowledge base was still intact and he used it quite skilfully during the problem structuring phase. However, the patient's problem-solving behaviour differed from the control's behaviour in the following ways: (1) he was unable to make the transition from problem structuring to problem solving; (2) as a result preliminary design did not start until two thirds of the way into the session; (3) the preliminary design phase was minimal and erratic, consisting of three independently generated fragments; (4) there was no progression or lateral development of these fragments; (5) there was no carryover of abstract information into the preliminary design or later phases, and (6) the patient did not make it to the detailing phase. This suggests that the key to understanding our patient's deficit is to understand the cognitive processes and mechanisms involved in the preliminary design phase.

We appeal to a theory of design problem solving (Goel, 1995) that associates cognitive processes involved in preliminary design with "lateral" state transformations and argues that "ill-structured" representational and computational systems are necessary to support these transformations. We conclude that the neural basis of this system is selectively damaged in our patient.
\end{abstract}

\section{INTRODUCTION}

Planning deficits have long been associated with frontal lobe lesions (Grafman, 1989, 1995; Shallice, 1982, 1988; Spector \& Grafman, 1994; Stuss \& Benson, 1986). Harlow $(1848,1868)$ talks about Phinias Gage "devising many plans of future operation but discarding them before they were executed...”. Penfield relates the story of his sister who, after the removal of her right frontal lobe, was unable to organize a dinner (Penfield \& Evans, 1935).

Such clinical observations are compelling but anecdotal. There are very few tasks/studies in the experimental neuropsychology literature that can be said to tap planning/design abilities. The most

Requests for reprints should be addressed to Vinod Goel, Department of Psychology, York University, 4700 Keele St., Toronto, Ontario, Canada, M3J 1P3, or to Jordan Grafman, Cognitive Neuroscience Section, National Institute of Neurological Disorders and Stroke, National Institutes of Health, Bethesda, Maryland, USA.

The first author is indebted to Professor Tim Shallice for hosting him at the Institute of Cognitive Neuroscience during the 199798 year, and for advice and encouragement during the writing of this paper. We are also grateful to Dr Paul Burgess and two anonymous reviewers of this journal for advice and suggestions that improved the manuscript. This work was supported in part by a York University Faculty of Arts Fellowship and NSERC and SSHRC grants to the first author. 
widely used task for such purposes is the Tower of Hanoi task. We have argued that while the Tower of Hanoi is an interesting task, it is not an optimal task to study planning - certainly not planning in real-world situations (Goel \& Grafman, 1995). It is in ill-structured real-world situations that patient planning deficits are most likely to emerge.

The ill-structured and well-structured distinction can be traced to Reitman (1964). Reitman classified problems based on the amount of information available in the problem vector (start state, goal state, and transformation function). The Tower of Hanoi is a typical example of a well-structured problem. In such puzzles, the start state is completely specified, as is the goal state and the set of legal transformations (though generating or selecting the "best" transformation at any given point may be a difficult task). Planning a meal for a guest is an example of an ill-structured task. The start state is incompletely specified (e.g., How hungry will they be? How much time and effort do I want to expend? etc.) The goal state is also incompletely specified (e.g., How much do I care about impressing the guest? Should there be three or four courses? Would salmon be appropriate? Would they prefer a barbecue or an indoor meal? etc.). And finally, the transformation function is also incompletely specified (e.g., Should I have the meal catered, prepare it myself, or ask everyone to bring a dish? If I prepare it, should I use fresh or frozen salmon? etc.). This is an important distinction in the cognitive science problem-solving literature and is further developed in Goel (1995).

Recently a number of researchers have moved beyond well-structured neuropsychological tests and tried to approximate real-world situations (Bechara, Damasio, Damasio, \& Anderson, 1994; Goel, Grafman, Tajik, Gana, \& Danto, 1997; Shallice \& Burgess, 1991a, 1991b). The Goel et al. study is of particular methodological relevance. They studied 10 frontal lobe patients and 10 normal controls engaged in a real-world financial planning task and used a "verbal protocol analysis" methodology (Ericsson \& Simon, 1993)-developed in the cognitive science literature - to quantify and analyse the complex data generated by the task. Among their findings were the following: patient perfor- mance was impoverished at a global level but not at a local level; patients had difficulty in organising and structuring their problem space; and once patients began problem solving, they had difficulty in allocating adequate effort to each problem-solving phase.

In the present report we follow up the group study with a detailed case study of a professional architect with a right prefrontal cortex lesion. Architects plan and design for a living. We approximated a real-world architectural planning task by requiring the patient to develop a new design proposal for our lab space and utilised a verbal protocol analysis methodology to analyse the data. Our results are consistent with the group study and go beyond it in a number of ways. The key to understanding our patient's deficit in this planning task is to understand the cognitive processes and mechanisms involved in the preliminary design problemsolving phase. The case study approach, combined with verbal protocol analysis, allows us to examine these processes in some detail. We appeal to a theory of design problem solving (Goel, 1995) that associates cognitive processes involved in preliminary planning with "lateral" state transformations and argues that "ill-structured" representational and computational systems are necessary to support these transformations. We conclude that the neural basis of this system is selectively damaged in our patient.

\section{METHOD}

\section{Subjects}

\section{Patient history}

The patient, PF, was a right-handed 57-year-old Caucasian male architect (with 17 years of education). In October 1984 PF had a grand mal seizure, was treated for a stroke, and hospitalised for 3 days. In 1985 he was diagnosed and treated for a right frontal parietal parasagittal meningioma. The patient underwent four resections (the first one in 1985) and was treated with one 6-week course of radiation (1991). MRI scans taken at NIH in August 1994 are reproduced in Figure 1. They 

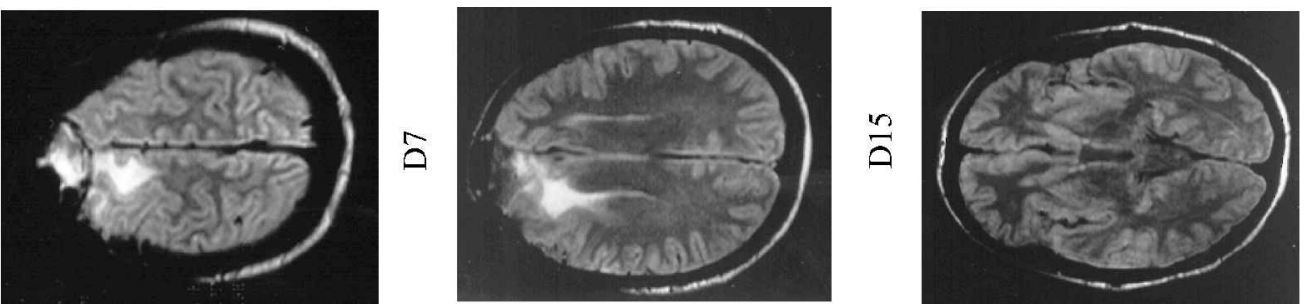

กิ
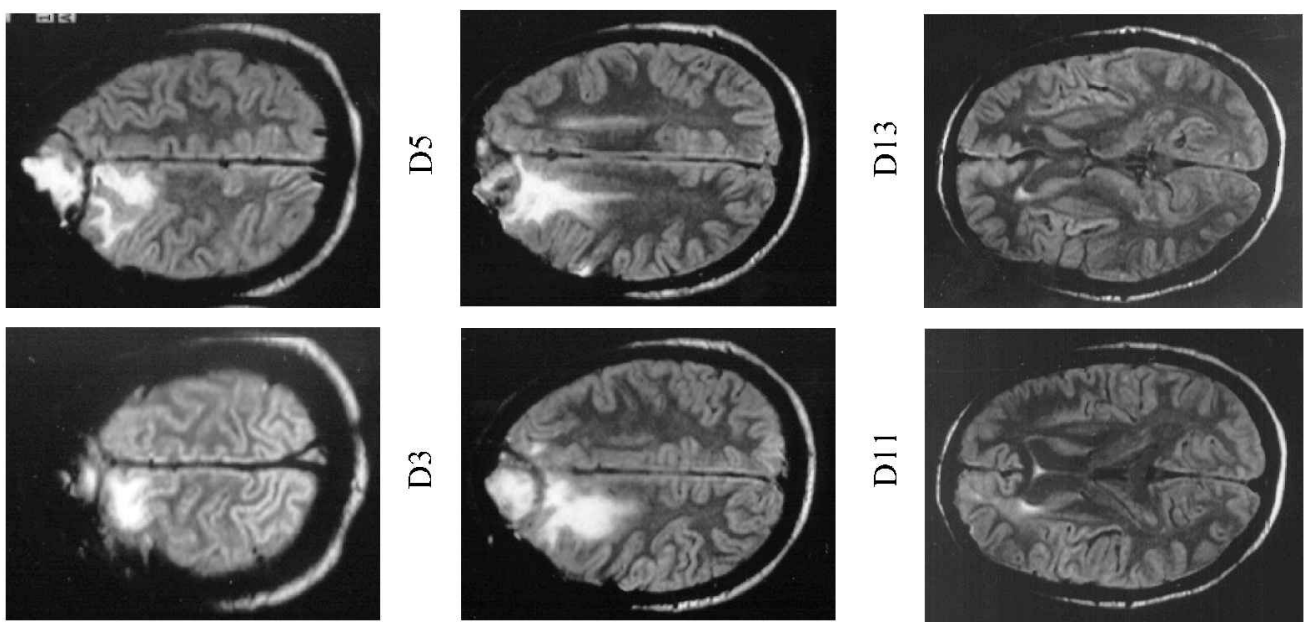

$\widetilde{2}$
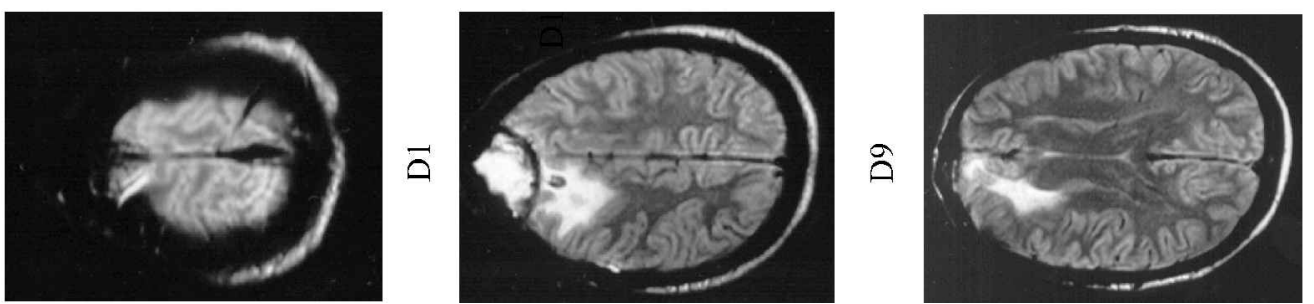

$\frac{a}{a}$

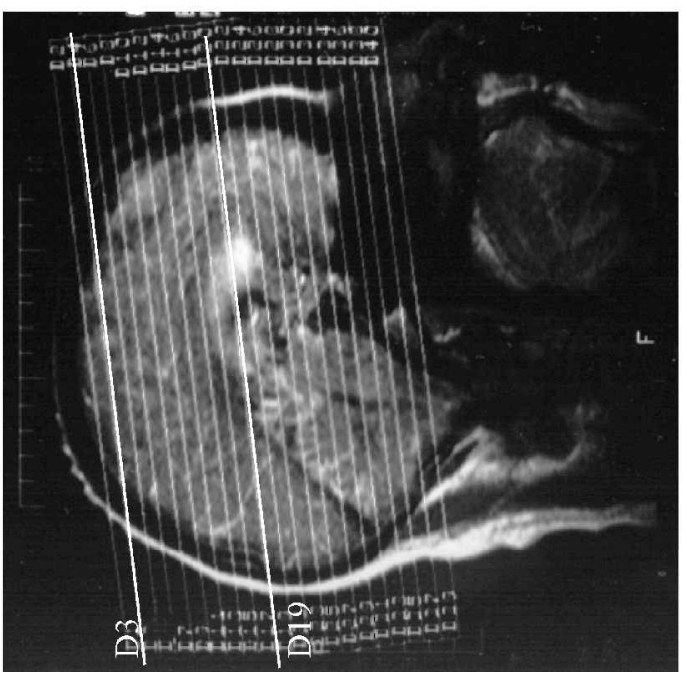

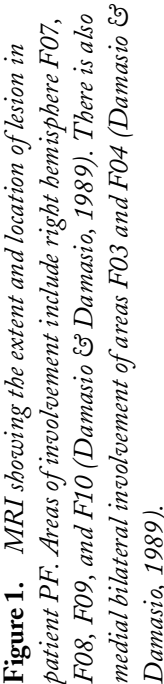


show a predominantly right-hemisphere lesion to the prefrontal cortex (including areas F07, F08, F09, and F10) (Damasio \& Damasio, 1989). There is also medial bilateral involvement of areas F03 and F04 (Damasio \& Damasio, 1989). The patient reported a "nervous breakdown" at the age of 19; otherwise his previous medical history was uneventful.

$\mathrm{PF}$ earned a graduate degree in Architecture from Yale University. He scored in the 98th percentile on the Graduate Record Examination in math and science. After graduation he practiced in the USA until the mid-1970s and then moved to Spain to work on a long-term "dream project". He was successfully self-employed in Spain until 1985 when he returned to the USA due to his illness. When we tested him he was involuntarily retired and lived at home with his mother.

\section{Current evaluation}

Testing observations. PF was tested at NIH in 1993. He was highly motivated and attempted to complete our testing in a timely fashion. He called often to schedule appointments, to reconfirm appointments, and came to the NIH with his records, waning to discuss not only the study he was participating in but other studies at the NIH. He would bring handwritten notes about his behaviour between sessions, along with comments on his behaviour during the previous session, as well as articles he found in newspapers and scientific journals.

PF was occasionally impatient with test instruction (all instructions would eventually be given and understood). He was sometimes obsessive about irrelevant items or problems and unable to organise some of his activities to optimally achieve a goal (like the paperwork necessary to get on disability). At the same time, he felt he was more hypergraphic, religious, less interested in sex, and less connected to his feeling of self. He gave excessive details about his sex life when asked by the examiner. He could remember the order of his life events before the tumour but afterwards had a much harder time organising and retrieving autobiographical events. He had a poor sense of time, was apathetic, and complained that his memory was not as good as it used to be. PF was forced to retire due to an "inability to design". His ability to draw was intact so he was attempting to get back into the work force as a draftsman doing "as-built drawings" (i.e., drawings/documentation of buildings as they are actually constructed).

During testing PF experienced depression and fatigue. PF's Beck Depression score was $25^{1}$. Testing sessions were scheduled for half days to minimise fatigue. Two examiners noted some frustration and anger with computer-administered tests.

As can be seen in Table 1, PF's Wechsler Adult Intelligence Scale-Revised (WAIS-R) (Wechsler, 1981) scores were in the superior range at the time of testing and his Wechsler Memory Scale-Revised (WMS-R) (Wechsler, 1987) scores were above average. On the WAIS-R when asked who Martin Luther King was, he replied that "he was a black religious leader who was killed for boycotting, possessed a rallying spirit, delivered the 'I Have a Dream speech'. ..." On the Twenty Questions test (Lezak, 1995), he solved the problems efficiently by starting with large categories (e.g., is it a living thing?) and narrowing down options with constraint-seeking questions. In conversation he was exceptionally intelligent and articulate and dis-

Table 1. $P F^{\prime}$ s scores on $I Q$ and memory tests

\begin{tabular}{ll}
\hline Test & Score \\
\hline$W A I S-R$ & \\
Full & 125 \\
Verbal & 120 \\
Performance & 128 \\
& \\
$W M S-R$ & \\
General & 113 \\
Verbal & 100 \\
Visual & 106 \\
Attention/Concentration & 111 \\
Delayed recall & 106 \\
\hline
\end{tabular}

\footnotetext{
${ }^{1}$ When patients are depressed, their performance shows less effort and imaging studies indicate left frontal hypometabolism. Our patient was depressed and it is possible that his performance may have been affected by his depression.
} 
played an excellent vocabulary. On the Controlled Oral Word Association (COWA) task he generated 73 words for "FAS" (Spreen \& Strauss, 1991) with only 2 repetitions and 2 rule violations; he named 56 items on the Boston Naming Test (BNT) (Kaplan, Goodglass, \& Weintraub, 1983), and his National Adult Reading Test (NART) (Nelson, 1982) error score was 14 (1 semantic and 13 phonologic errors). On the Wisconsin Card Sorting Test (WCST) (Heaton, Chelune, \& Talley, 1993) he completed all 6 categories in 77 trials, making only 8 perseverative and 2 nonperseverative errors. His performance on the Stroop Colour Word Test (Jensen \& Rohwer, 1966) was within normal limits. He successfully solved 14 out of 14 Tower of London (Shallice, 1982) problems without any rule violations. However, he experienced difficulty with a (computeradministered) Tower of Hanoi task (Goel \& Grafman, 1995; Reeves, Kane, \& Winter, 1994; Samet \& Marshall-Mies, 1987). He successfully completed only three of nine problems within the allowed time ${ }^{2}$. Yet despite his exceptional neuropsychological profile, he was involuntarily unemployed and unable to function as an architect.

We matched the patient for age and education with a 54-year-old architect and engaged both in a simple architectural design/planing task ${ }^{3}$. The control architect was a professor of architecture at the University of Maryland and had experience in private practice. He reported no neurological or psychiatric history.

\section{Task}

Given that the patient was an accomplished architect, and the issue of interest is the role of the prefrontal cortex in planning and design problem solving, we devised and administered a planning/ design architectural task.

Both subjects were asked to propose a redesign for our lab space. They were given the following problem statement specifying a set of constraints and encouraged to ask for additional information as necessary:

Our lab space is located in Room 5D51. It currently houses three scientists and five research assistants. Another scientist is expected in January. The number of research assistants can increase up to 16 during the summer months.

The space is used for reading, writing, computing, telephone conversations, and so on. In fact, we do all of our work in this space except for seeing patients. Some of us spend up to 10 hours per day there. It is a very dismal environment.

Your task is to reorganise, and redesign the space such as to increase our comfort and productivity. We do not have a budget for the redesign. However, we do have the option of exchanging some of our furniture at the surplus store, and perhaps we can pool personal time and resources to do some painting and cleaning.

You have 2 hours to propose a design. You may spend up to 15 minutes of the first hour in the lab space. While there, you may measure, make notes and sketches, and ask anyone there any questions you think relevant. You may revisit the lab for $10 \mathrm{~min}-$ utes anytime during the second hour. Please begin.

Both of our subjects had experience designing office spaces and noted that this was a very easy problem for them. The manner in which we administered the task was also familiar to our subjects. They commented that it corresponded to the 2hour design studio skits that are a part of an architect's training.

\section{Data collection}

Subjects were brought into a testing room and presented with the above task. They were given written instructions that explained the experimental procedure. In these instructions subjects were asked to

\footnotetext{
${ }^{2}$ Given his aversion to computers, his poor performance may simply have been a function of the fact that the task was computer administered (see earlier). When given the task in a non-computer format he was able to solve (difficult) five disk problems.

${ }^{3}$ The experiment was actually set-up with two controls, the expert architect whose data we report, and an age- and educationmatched nonarchitect (a 55-year-old lawyer). We used the nonarchitect to control for the possibility that our patient's architectural knowledge base had been damaged and his behaviour had reverted to that of a novice. Given that this was clearly not the case, and that the presentation/introduction of the novice control data introduces issues of expert/novice differences that complicate and lengthen the paper without adding additional insight into our patient's behaviour, we have decided not to incorporate this data into the paper. We briefly summarise the novice control's performance in Footnote 5.
} 
talk aloud as they proceeded through the task. The sessions were video taped. Subjects were asked not to try to explain what they were thinking but, but rather just to vocalise the fragments of thoughts and ideas they might be attending to at that time. They were told that time was not a critical factor but it was important that they fully engaged the task. They were also told that the information contained in the problem scenario was incomplete and were encouraged to ask questions as necessary. The experimenter was present in the room and answered any questions the subjects asked, but he/ she did not initiate questions or discussion. Subjects had access to pen and paper and other drawing aids.

The video recordings, along with anything the subjects wrote, constituted the raw data. They are clearly very complex data. In analysing them, we were interested in not only how well subjects performed the task, but more importantly, how they performed the task (i.e., their cognitive strategies and processes).

\section{Data analysis}

The accepted way of analysing such data is with a methodology called "protocol analysis" (Ericsson \& Simon, 1984). It is tailored to such complex data and allows for both qualitative and quantitative analysis. Most results concerning complex human problem solving in the cognitive science literature stem from a combination of this methodology and Information Processing Theory (Newell, 1990; Newell \& Simon, 1972).

Protocol analysis treats verbal data like any other behavioural data. At the top level subjects are generating noise/sound waves. We interpret these "phonetic acts" as "phatic acts," that is, as sentences of a natural language. Furthermore, we freely assign meaning to these sentences. All this is prior to any explicit analysis. We can do this freely because we believe the utterances to be meaningful and understand their meaning by virtue of belonging to the same linguistic community as subjects. The explicit analysis and interpretation of the data beings with the transcription of the verbalisations. At this point there is some pretheoretical preprocessing that involves the filtering of facial gestures, hand wav- ing, intonation, etc. The preprocessed transcribed text is correlated with the written material, and coded. The coding can be with a priori categories, or the understanding of the verbalisation and formation of categories can occur in parallel. In either case the vocabulary/categories are given by a theory.

A common misconception of the methodology is to regard is as a form of introspection. Note, however, that in instructing subjects, one is not asking them to tell what they are doing or thinking. It is the theorist's job to figure this out. What one is asking subjects to do is to verbalise whatever thought contents they are attending to as they do the task. The accepted interpretation of these verbalisations-within information processing theory - is that they give us a trace of the contents of the subject's activated memory structures. From this trace the theorist infers the operations the subject applied to these contents, and the controlstrategies that guided the subjects through the problem space. A number of studies have indicated that the demands of such verbalisations may affect the speed of problem solving but otherwise do not affect performance (Ericsson \& Simon, 1984). Goel et al. (1997) have demonstrated that frontal lobe patients are capable of giving verbal protocol during problem solving.

\section{Coding scheme}

The verbal protocols were first transcribed and cross-referenced with the written and drawn documents. The transcribed protocols were divided into utterances or statements, and each statement was coded with a three-level scheme developed by Goel and Pirolli (Goel, 1994, 1995; Goel \& Pirolli, 1992). The first two categories were determined by a theory of design problem solving (Goel, 1994, 1995; Goel \& Pirolli, 1992). The third category was determined by Information Processing Theory (Newell, 1990; Newell \& Simon, 1972). Each level of the scheme is associated with a different vocabulary and granularity, and provides insight into a different aspect of the subject's cognitive process.

The first step in the coding process involved breaking the protocols into individual statements representing single "thoughts" or ideas. Content 


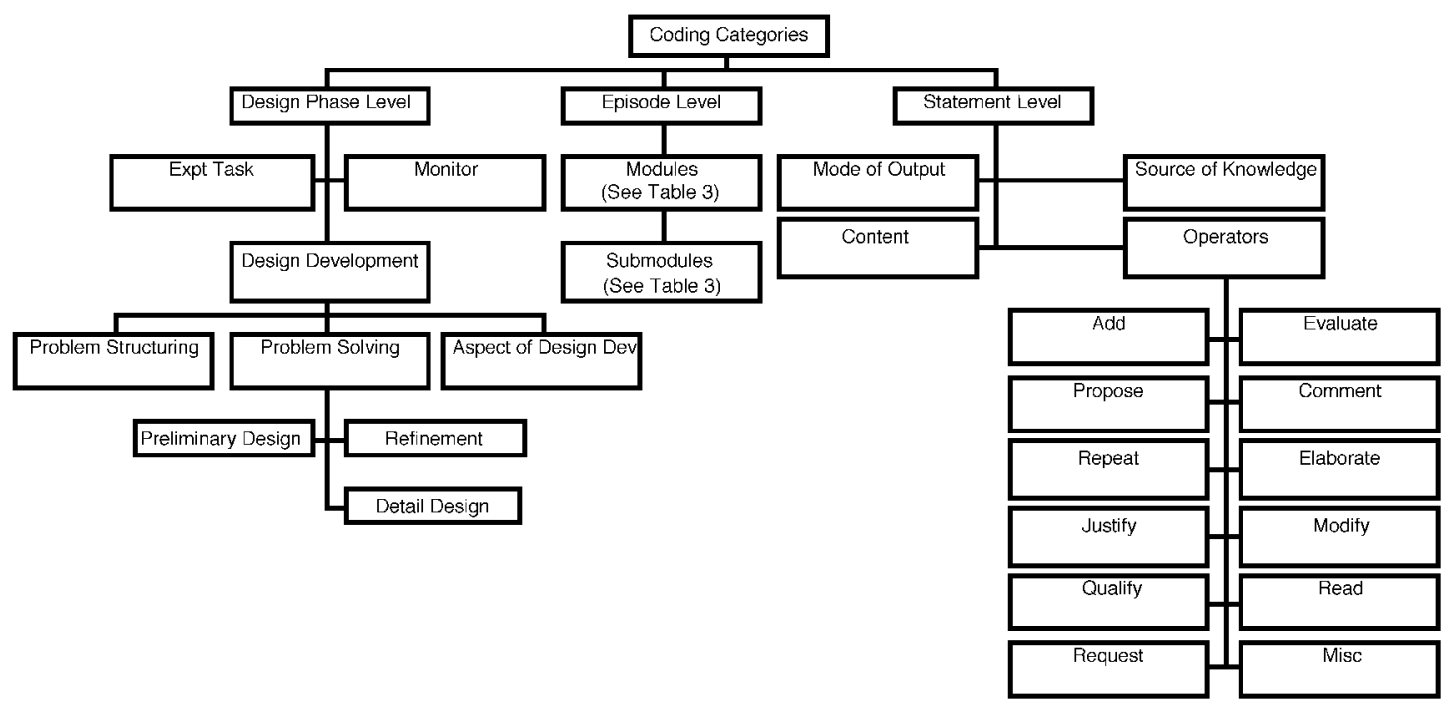

Figure 2. Specific coding categories.

cues, syntactic cues, and pauses were used to effect this individuation. The vocabulary at this level consists of states, operators, and evaluation functions and comes straight from information-processing theory. We took each statement as constituting a state in the subject's problem space and inferred the operator applied to it. We found the following 12 operators adequate for our purposes: add, evaluate, propose, comment, repeat, elaborate, justify, modify, qualify, read, request, and misc. This level of analysis gives a picture of cognitive processes at the granularity of a few seconds (see Appendix A for details).

These statements were then aggregated into $e p i-$ sodes, which are connected sequences of statements in the service of a common subgoal. In our architectural planning task, episodes were organised around "modules" or subproblems. Modules are taskspecific and therefore vary from problem to problem. They are largely determined by an interaction between problems and the background knowledge and practices of subjects. Our subjects broke down the problem into 9 modules (subproblems) and 47 submodules, as itemised in Table 3. Episodes typically have a duration of 1 to 2 minutes. The vocabulary at this level is one of goals, subgoals, and strategies.
Episodes were further aggregated into a design phase level. The design phase level consists of experimental task statements, monitor statements, and design development statements. The design development statements were further divided into problem structuring and problem solving phases. Problem structuring is a necessary prerequisite for the solution of ill-structured problems. It involves generating information missing from the problem scenario so that the problem space can be constructed. Once the problem space is specified, problem solving can begin. Problem solving was further differentiated into preliminary design, refinement, and detailing. Preliminary design statements result in the initial generation and exploration of ideas. Refinement statements serve to elaborate and develop an idea. Detailing statements specify the final form of an idea. These phases typically have durations of 1 to 10 minutes, and are generally engaged sequentially, starting with preliminary planning, passing through refinement, and ending with detailing. However, it is common for subjects to return to an earlier phase as previously unnoticed aspects emerge. The specifics of these coding categories are summarised in Figure 2 and discussed more fully in Appendix A. 
Drawings made by the subjects were used as states in a macro-level reconstruction of their respective problem spaces (see Figures 5 and 6). The individuation of drawings and sketches was reasonably straightforward. For the most part, the subjects individuated the output by drawing rectangles around each separate drawing and even numbering them. Often they assigned a linguistic label to each drawing, which helped in the individuation process. Given the state space, we then inferred the type of transformation that was applied to get from one state to another.

Transformations were classified as either new generations or transformations of previous solutions. New generation result in new drawings unrelated to previous drawings. At the syntactic level a new generation means that the marks which constitute the drawing are unrelated in form to marks constituting earlier drawings. At the semantic level it signifies that the content of the current drawing is unrelated to the content of earlier drawings. Most drawings/ states are transformations of previous solutions or drawings. Three such transformations are recognised: lateral transformations, vertical transformations, and duplication. A lateral transformation modifies a drawing into another related but distinctly different drawing (as opposed to a more detailed version of the same drawing, a totally unrelated drawing, or an identical drawing). A vertical transformation reiterates and reinforces an existing drawing through explication and detailing. A duplication transformation results in movement from a drawing to a type identical drawing. Syntactic transformations relate the form of the marks that constitute drawings whereas semantic transformations relate the associated contents or ideas.

The objectivity of the methodology lies in the fact that, once the categories have been developed and their recognition criteria explicated, different individuals can apply the categories to the data with similar results. The data were initially coded by the first author. They were then recoded (blind) by a research assistant unfamiliar with the project. There was a mean of $87 \%$ agreement between the two coders. The disagreements were resolved through augmentation. Both coders agreed on the results reported here.

\section{RESULTS}

As in previous frontal lobe patient studies using protocol analysis (Goel et al., 1997) we found that $\mathrm{PF}$ was quite able to give a verbal protocol during the problem-solving process. The problem-solving time varied from 95 minutes for the patient to 90 minutes for the control. The patient generated 945 statements for a mean of 9.9 statements per minute. The expert control generated 1279 statements during a 90-minute session for a mean of 14.2 statements per minute.

The coding scheme analyses the data at three different levels of granularity. It should be noted that at each level of analysis, the performance of the expert control is very typical of designers solving such problems, in such circumstances (Goel \& Pirolli, 1992).

At the design level (see Table 2), there is considerable dissimilarity in the allocation of cognitive effort (as measured by the number of statements) between the patient and control. The control subject allocates roughly $93 \%$ of his statements to the problem-solving effort (design development), 3\% to understanding and clarifying the experimental procedure, $3 \%$ to monitoring progress, and $2 \%$ to miscellaneous categories. In contrast, the patient spends only $70 \%$ of his statements advancing the problem-solving effort. A further $8 \%$ of his statements are used to understand and clarify the experimental procedure, and $10 \%$ are used in selfmonitoring progress. A full $12 \%$ of the patient's statements fall into the misc category, and do not advance the problem space.

The patient's increased number of clarification of task statements is due to the fact that he engaged the experimenter in a discussion of the talk-aloud aspect of the methodology. He thought (as do many normal controls) that we were asking him to explain his mental processes. But once this was clarified he was able to proceed with the task. This clarification occurred at the beginning of the experiment. There was no need to revisit the experiment instructions during the task.

The patient also had an increased number of self-monitoring and misc statements. Most of these statements occurring during points of particular 
difficulty, like the preliminary design phase. The self-monitoring statements were used to explicitly monitor and control problem-solving behaviour (e.g., "I know what I want to draw, but I just don't do it. It's crazy. Well, force yourself, Peter. Force yourself", and "But I'm making excuses. I'm not dealing with it like-like I-like I normally would.”) The misc statements were triggered by similar difficulties. Many of the misc statements were unsolicited explanations of the causes of these difficulties (e.g., "But it's as if I'm getting a train of thought and then I start to draw it, and then I lose the train of thought. And, then, I have another train of thought that's in a different direction, and the two don't ... and this is a very simple problem.") Other misc statements were tangential remarks triggered by the task, but did not advance the problem-solving effort (e.g., "I stopped at an Inn and the guy had a problem much like this, and I had a goal in my mind what to do with the space and I was clear. It had to do with seeing a view. But then once I had to think about something else, like the heights of the heads and what - and the porch that was outside, I was just stopped cold. ...”)

Within the design-development phase the patient's allocation of statements between problem structuring and problem solving was also quite different from the expert control. The control spent $29 \%$ of his statements structuring the problem and $71 \%$ engaged in problem solving. The patient spent $66 \%$ of design development statements on problem structuring and $34 \%$ on problem solving. This

Table 2. Design level statements

\begin{tabular}{lcc}
\hline & $\begin{array}{c}\text { Expert Control } \\
\%\end{array}$ & $\begin{array}{c}\text { Patient PF } \\
\%\end{array}$ \\
\hline Experimental task & 3 & 8 \\
Self-monitoring & 3 & 10 \\
Design-development & 93 & 70 \\
Problem-structuring & 26 & 46 \\
Problem-solving & 66 & 24 \\
$\quad$ Preliminary design & 19 & 12 \\
Refinement & 27 & 12 \\
Detailing & 21 & 0 \\
& & \\
Misc & 2 & 12 \\
\hline
\end{tabular}

pattern of allocation of design-development statements is the same as reported in a recent group study of planning in frontal lobe patients (Goel et al., 1997).

Within the problem-solving phase the control subject allocated roughly equivalent effort to the preliminary, refinement, and detailing phases of the design (Table 2). The patient confined his statements to preliminary design and refinement (Table 2). Again, this distribution of statements resembles that reported in Goel et al.'s (1997) group study of planning.

Figures 3 and 4 show the temporal distribution of design-level statements for the control and patient, aggregated over 5-minute intervals. The control's temporal distribution of statements is quite typical of designers (Goel, 1995). He began by problem structuring and had it largely completed in the first quarter of the problem-solving session. The next part of the session was devoted to preliminary design, followed by design refinement and then detailing, each in roughly equal proportion. The whole process was accompanied by some selfmonitoring and the phases reoccurred as needed throughout the session.

The patient's progress through the problem space was quite different from the control's. The patient spent most (two thirds) of the session in problem-structuring mode. The preliminary design phase occurred at the tail end of the session. Its duration was short; it was accompanied by much self-monitoring and a great many misc statements; and as we will see shortly, the subject's movement through this phase was erratic and lacked progression. It was a phase with which the patient had enormous difficulty. At the 87-minute mark, the beginning of the refinement period, the patient noted "let's start again." Refinement of this proposal occurred during the last few minutes and the session ended abruptly.

Overlaid on top of the temporal distribution of design-level statements in Figures 3 and 4 are the key drawings made by the subjects and some highlights of the session. Drawings depict the generation and development of ideas/concepts. As such they constitute states in the problem space. Figures 5 and 6 show the actual drawings made by the 

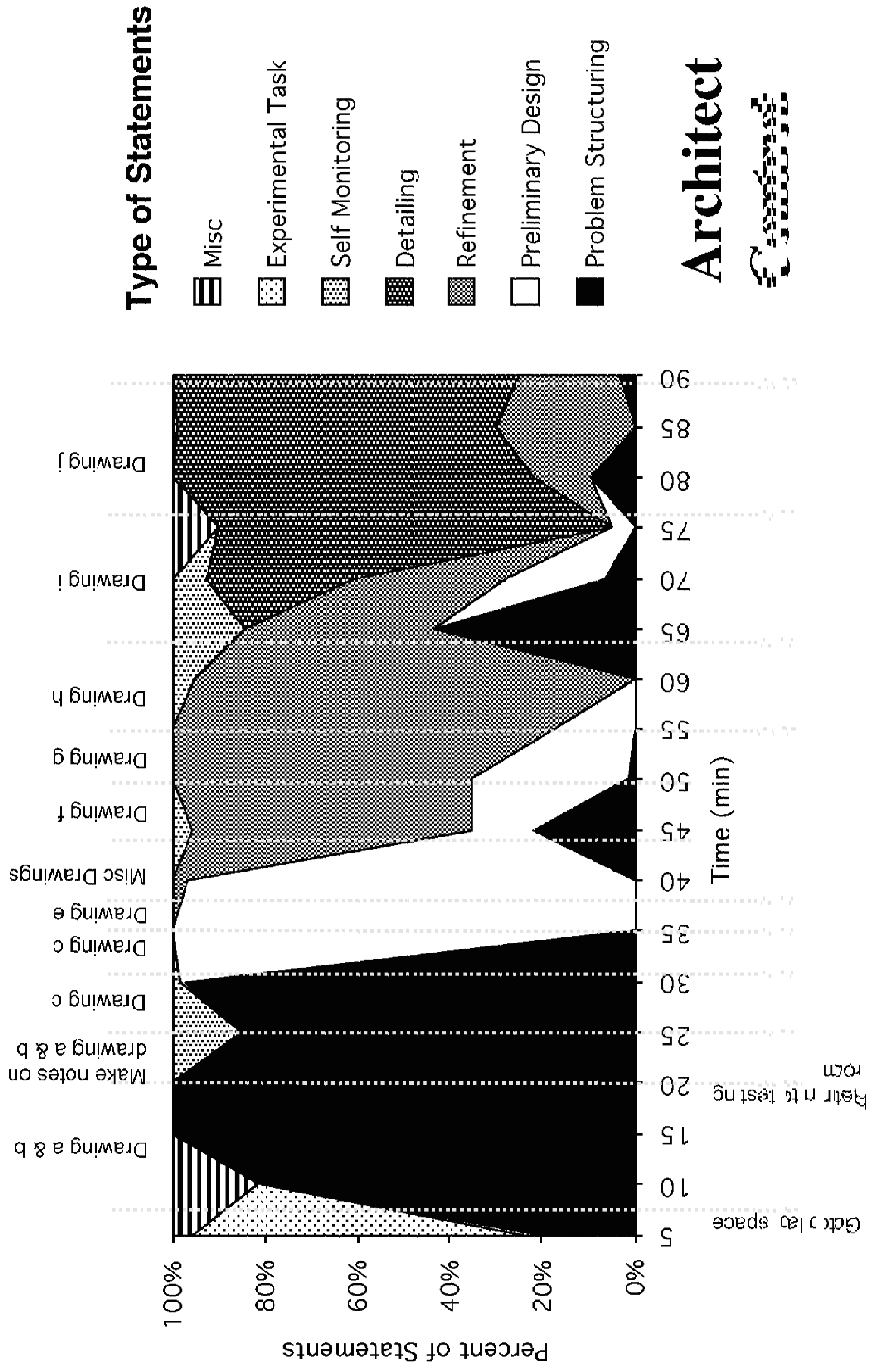

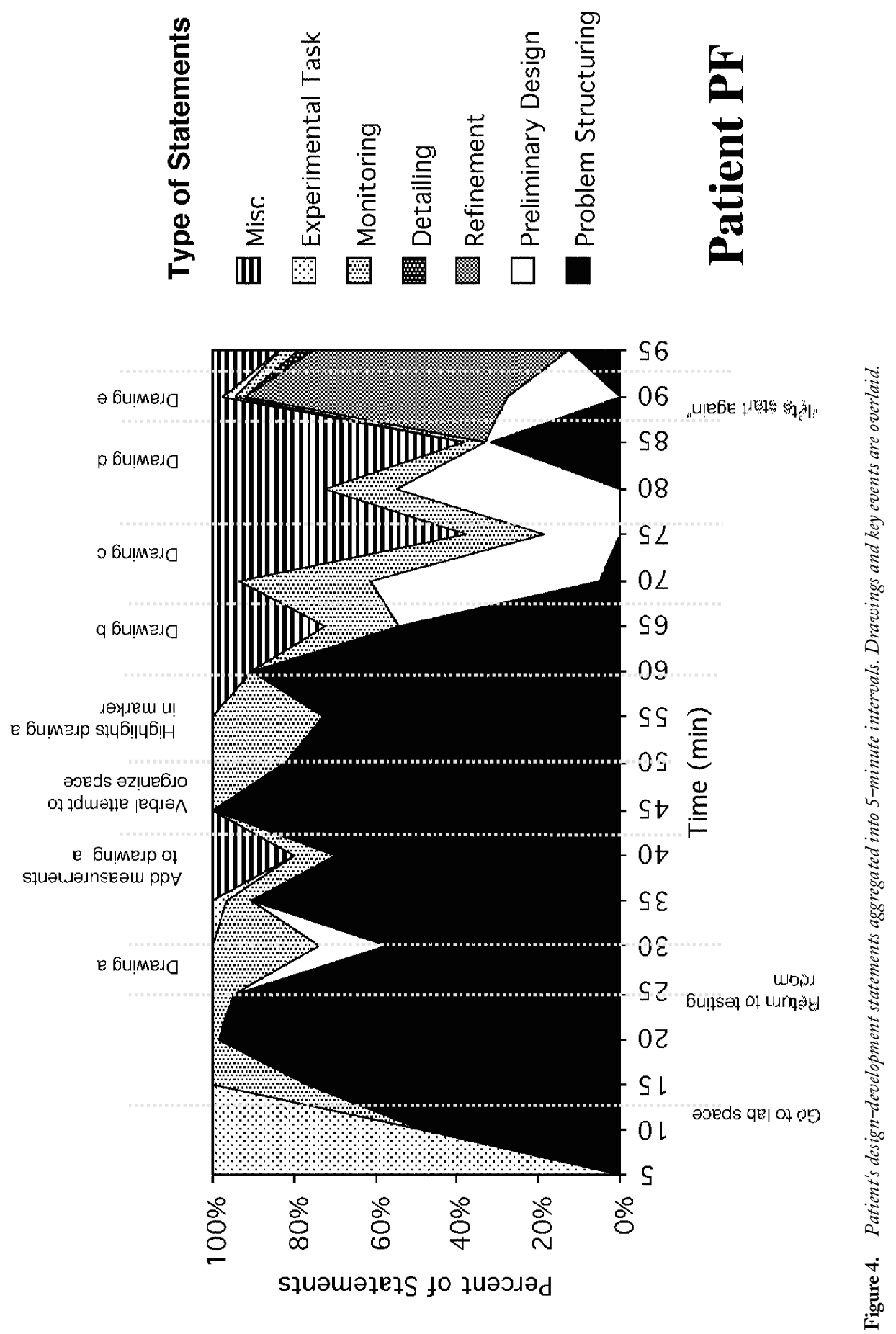
subjects and the type of transformations that lead from one drawing/idea to the other.

There are nine states in the control's problem space (Figure 5): a start state (a \& b), a goal state ( $j$ ) and seven intermediate states $(\mathrm{c}-\mathrm{i})$. The starting state for the control is drawing (a) of the existing lab space and the accompanying measurement drawing (b). Both drawings were made in the lab space. Three of the intermediate states belong to the preliminary design phase (c-e); four belong to the refinement phase $(\mathrm{f}-\mathrm{i})$; and the final state belongs to detailing $(\mathrm{j})$. The preliminary design states are all quite abstract. He began by considering "circulation patterns" in drawing (c). This pattern constitutes his kernel idea. It was developed and transformed to deal with the issue of "social organization" (d) and "permanent and transient spaces" (e). The refinement drawings are structural. They depict workstations, tables, doors, windows, and corridors. The subject transformed state (e) into a proposal (drawing $\mathrm{f}$ ) halfway through the session that he considers "reasonable". However he thought the centre condition could be improved. $\mathrm{He}$ therefore held the perimeter conditions constant and transformed the centre in drawing (h). He rejected drawing (h), returned to drawing $(\mathrm{f})$ and transformed it into drawing (g). He was happy with the idea depicted by drawing $(\mathrm{g})$. He then shifted gears and began to detail and fine-tune the proposal, first in section (drawing i) and then in plan (drawing $\mathrm{j}$ ).

The movement from states $(\mathrm{c})$ to $(\mathrm{g})$ is underwritten by lateral transformations. A lateral transformation is one where movement is from one idea to a slightly different idea rather than a more detailed version of the same idea (Goel, 1995). The transformation of state (g) to (i) and from (i) to (j) is underwritten by vertical transformations. A vertical transformation is one where movement is from one idea to a more detailed version of the same idea (Goel, 1995). The control's state space contains five lateral transformations (LT) and two vertical transformations (VT).

An analysis of the patient's state space tells a very different story. It contains five states (Figure 6): a start state (a), a goal state (e), and three intermediate states $(\mathrm{b}-\mathrm{d})$. The start state drawing of the exist- ing lab space (a) was completed by the patient from memory in the testing room. It is as detailed and accurate as the control's drawing. The patient's final state drawing (e) was completed during the refinement phase. The three intermediate drawings $(b-d)$ were completed during the preliminary design phase. The first of these drawings (c) - the kernel idea-occurred two thirds of the way into the session. Unlike the preliminary drawings of the control, the patient was concerned with arranging furniture right from the start. But perhaps the most dramatic difference between the patient and control is that the patient's three preliminary design drawings are fragmentary and unrelated.

Preliminary design sketches are, almost by definition, fragments of ideas. Designers do not generate several independent fragments and choose between them. They generate a single idea/fragment and develop it though transformations (lateral or vertical) to a point where it is complete and can be evaluated (Goel, 1995). The patient made several (successful) attempts to generate idea fragments. But he was unable to develop and explore these ideas through the application of lateral transformations. Each of his preliminary drawings must be treated as independent ideas/fragments. Indeed, he tried to articulate the difficulty he was experiencing as follows:

You see, normally, what I would have, even as a student, I'd bethere would be sketches on top of sketches. And I could-it would be progressive. Here I seem to be doing several different thoughts on the same piece of paper in the same place, and it's confusing me. So, instead of the one direction that I had at the beginning, I have three or four contradictory directions with not a kind of anchor to work from....

On generating drawing (d) 87 minutes into the session, he said "let's start again." The only idea/ drawing transformation he engaged in was from $(\mathrm{d})$ to (e). This is a vertical transformation where he fine-tuned the proposal. So his state space consists of three new generations and one vertical transformation (VT).

An episode-level analysis of the data provides a measure of goals/subgoals and strategies pursued by subjects. The average duration of an episode was 1.2 minutes for the control and 1.6 minutes for the patient. Unlike the above analysis, episodes are 

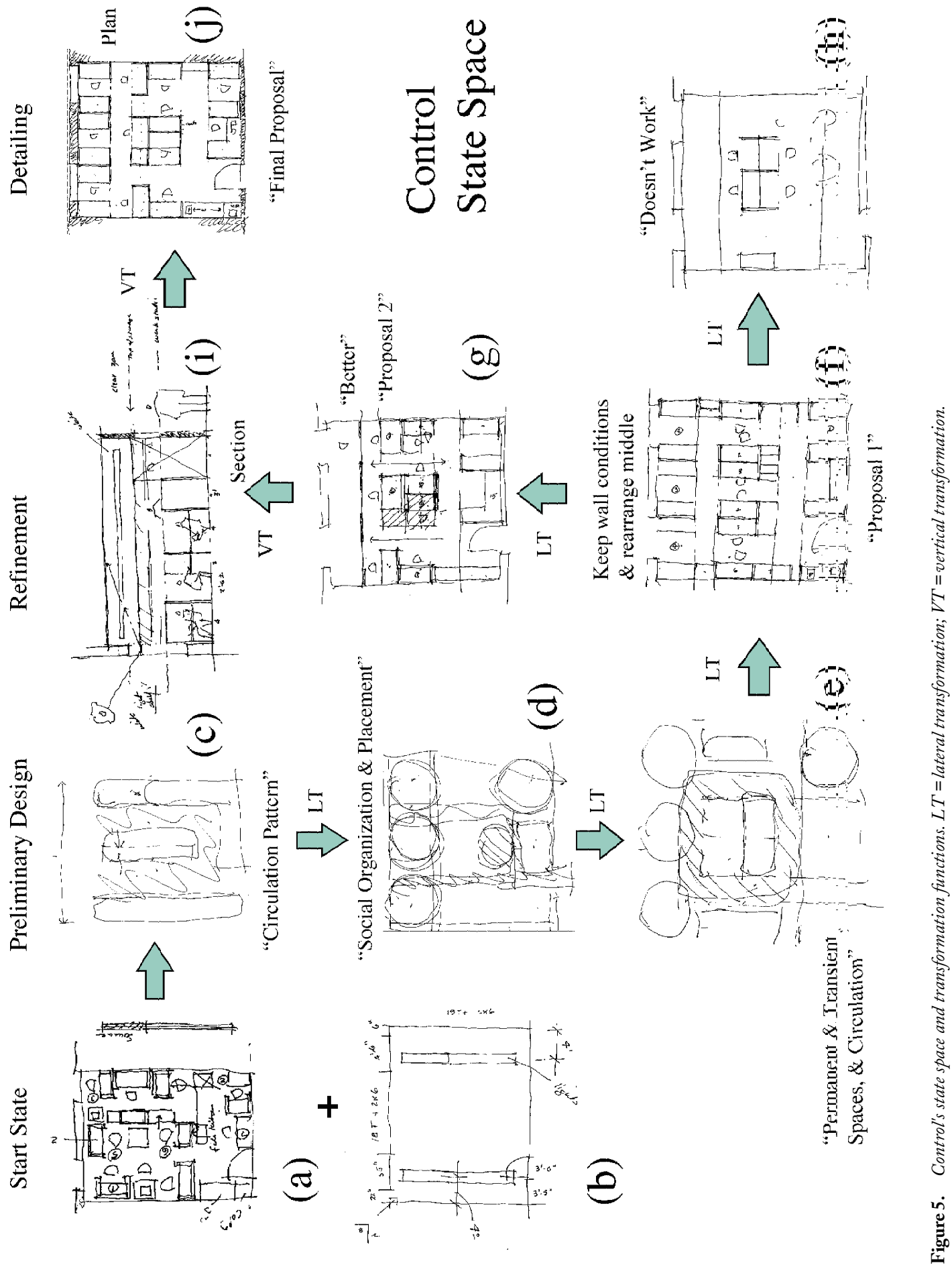


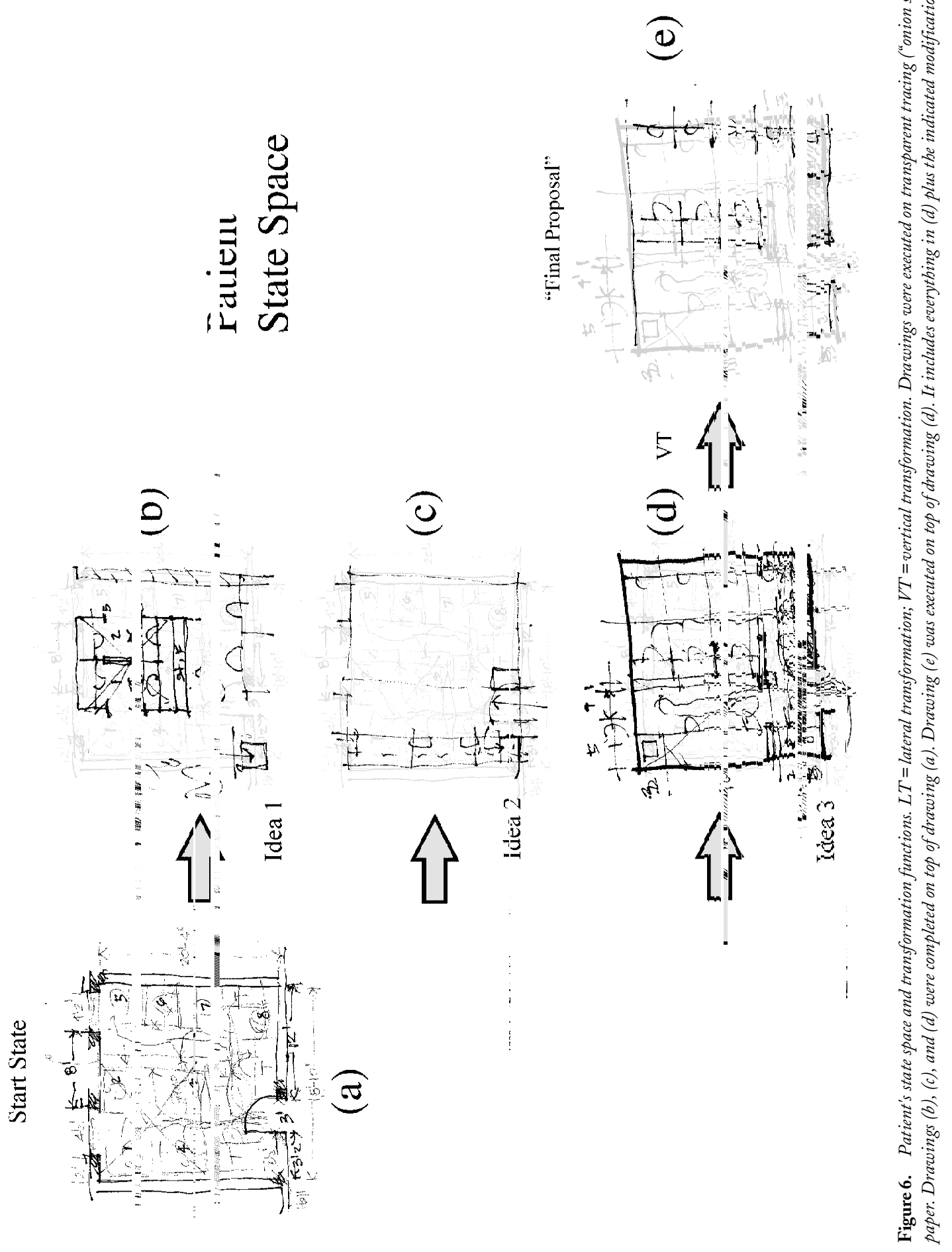


problem-specific; it is a content analysis. It gives an indication of the knowledge subjects bring to the problem space and the issues they consider. As can be seen in Table 3, the subjects considered issues ranging from information about users (e.g., numbers, categories), their goals (e.g., a quieter environment), behaviours/activities the users need to engage in (e.g., meetings, writing), the functions the artefact/space needs to support (e.g., circulation patterns), and the actual structure of the artefact (e.g., printers, workstations, dimensions), etc.

These episodes are organised into nine modules in Table 3. The patient considered eight modules, omitting only "lighting". The normal control also considered eight modules, omitting "cleaning/ maintenance." So both the control and patient displayed the sophisticated knowledge base one would expect from experienced architects. For example, both discussed "social clustering and hierarchy," "communal facilities," "circulation patterns," etc. Only an experienced architect could bring such vocabulary and issues to bear on the task (see Footnote 5). However, PF did not pursue some of the modules (e.g., "circulation patterns" and "border conditions") in as much detail as the normal control. As a consequence, the total number of subgoals considered by the patient was 40 compared to 54 for the control ${ }^{4}$.

Table 3. Itemisation of modules and submodules utilised by patient (PF) and control (NC)

\begin{tabular}{|c|c|c|c|c|c|}
\hline$P F$ & $N C$ & Modules/Submodules & $P F$ & $N C$ & Modules/Submodules \\
\hline $\mathrm{x}$ & $\mathrm{x}$ & Users & $\mathrm{x}$ & $\mathrm{x}$ & Communal facilities \& space \\
\hline $\mathrm{x}$ & $\mathrm{x}$ & numbers & $\mathrm{x}$ & $\mathrm{x}$ & furniture \\
\hline $\mathrm{x}$ & $\mathrm{x}$ & class/categories & $\mathrm{x}$ & $\mathrm{x}$ & filing cabinets \\
\hline $\mathrm{x}$ & $\mathrm{x}$ & relationship between users & $\mathrm{x}$ & $\mathrm{x}$ & misc equipment \\
\hline $\mathrm{x}$ & $\mathrm{x}$ & changes over course of year & $\mathrm{x}$ & $\mathrm{x}$ & printer \\
\hline $\mathrm{x}$ & $\mathrm{x}$ & work habits & & $\mathrm{x}$ & message board \\
\hline $\mathrm{x}$ & $\mathrm{x}$ & source of discomfort & & $\mathrm{x}$ & photocopying \\
\hline $\mathrm{x}$ & & Activities in space & $\mathrm{x}$ & $\mathrm{x}$ & closet space \\
\hline $\mathrm{x}$ & $\mathrm{x}$ & experiment design $\&$ analysis & $\mathrm{x}$ & $\mathrm{x}$ & storage space \\
\hline $\mathrm{x}$ & $\mathrm{x}$ & reading $\&$ writing & $\mathrm{x}$ & $\mathrm{x}$ & entrance zone \\
\hline $\mathrm{x}$ & $\mathrm{x}$ & meetings & $\mathrm{x}$ & $\mathrm{x}$ & dimensions \\
\hline $\mathrm{x}$ & $\mathrm{x}$ & Social concerns & $\mathrm{x}$ & $\mathrm{x}$ & configuration \\
\hline $\mathrm{x}$ & $\mathrm{x}$ & social clustering/social hierarchy & $\mathrm{x}$ & $\mathrm{x}$ & refrigerator \\
\hline $\mathrm{x}$ & $\mathrm{x}$ & community space & $\mathrm{x}$ & $\mathrm{x}$ & microwave \\
\hline $\mathrm{x}$ & $\mathrm{x}$ & private space & & $\mathrm{x}$ & Lighting \\
\hline \multirow[t]{2}{*}{$\mathrm{x}$} & $\mathrm{x}$ & character of space & & $\mathrm{x}$ & natural light \\
\hline & $\mathrm{x}$ & personalisation of space & & $\mathrm{x}$ & task lights/personal lamps \\
\hline $\mathrm{x}$ & $\mathrm{x}$ & noise levels & & $\mathrm{x}$ & fluorescent lights \\
\hline \multirow[t]{4}{*}{$\mathrm{x}$} & $\mathrm{x}$ & Circulation patterns & $\mathrm{x}$ & $\mathrm{x}$ & Border conditions \\
\hline & $\mathrm{x}$ & cul de sac & $\mathrm{x}$ & $\mathrm{x}$ & walls \\
\hline & $\mathrm{x}$ & dead space & & $\mathrm{x}$ & ceilings \\
\hline & $\mathrm{x}$ & congestion & & $\mathrm{x}$ & windows \\
\hline $\mathrm{x}$ & $\mathrm{x}$ & Private facilities \& space & $\mathrm{x}$ & $\mathrm{x}$ & door \\
\hline $\mathrm{x}$ & $\mathrm{x}$ & workstations & $\mathrm{x}$ & $\mathrm{x}$ & flooring \\
\hline \multirow[t]{2}{*}{$\mathrm{x}$} & $\mathrm{x}$ & computers & & $\mathrm{x}$ & venting \\
\hline & $\mathrm{x}$ & seating & & $\mathrm{x}$ & orientation $(\mathrm{N}, \mathrm{S}, \mathrm{E}, \mathrm{W})$ \\
\hline $\mathrm{x}$ & $\mathrm{x}$ & dimensions & $\mathrm{x}$ & $\mathrm{x}$ & dimensions \\
\hline $\mathrm{x}$ & $\mathrm{x}$ & configuration & $\mathrm{x}$ & & Cleaning/Maintenance \\
\hline
\end{tabular}

$\mathrm{x}=$ utilisation

${ }^{4}$ Given PF's excellent COWA task performance, it is unlikely that this difference in the number of categories is due to languagerelated problems such as mild dynamic aphasia. 
Further evidence of the patient's difficulties with the preliminary design phase is provided by analysing the aspects of the design development considered by the control and patient during the four phases of design development. Table 4 shows the percentage of design development statements (problem structuring, preliminary design, refinement, and detailing) the control and patient devote to the consideration of people (users), their goals/ purposes, the behaviours that need to be supported, the function the artefact needs to serve, and the structure of the artefact itself. In the case of the control one can see that during problem structuring and preliminary design the majority of the statements are devoted to abstract, nonstructural aspects of the design having to do with users, goals, behaviours, and functions. This distribution shifts with the refinement phase, where function and structure dominate, and again in the detailing phase, where structure dominates. For example, during the structuring phase the control considers issues of social hierarchy (research assistants vs. scientists). This is propagated and translated into structural arrangement in terms of giving the scientists more spacious workstations and placing them in corner locations.

The level of abstractness of the statements in the patient's problem-structuring phase is quite similar to that of the control. He solicits and generates much relevant information about people, purpose, and behaviours. The patient's refinement phase distribution is also not unlike the control's. However, the patient's preliminary design phase is dramatically different. Whereas the control carries the information regarding people, purpose, and behaviour through to the preliminary design phase (and in fact through to refinement and detailing) and uses it to guide the emerging design, the patient is unable to carry through this information and use it to guide problem solving.

The allocation of statements at the operator level appear in Table 5. The patient makes fewer written "statements" (or marks) than the control, suggesting a greater reliance on verbalisation, and decreased commitment to the generated ideas. The patient is also more reliant on the examiner and

Table 5. Distribution of operators, mode of output, and source of information

\begin{tabular}{|c|c|c|}
\hline & $\begin{array}{c}\text { Expert Control } \\
\%\end{array}$ & $\begin{array}{c}\text { Patient PF } \\
\%\end{array}$ \\
\hline \multicolumn{3}{|l|}{ Mode of output } \\
\hline Written & 31.96 & 15.44 \\
\hline Verbal & 68.59 & 83.96 \\
\hline \multicolumn{3}{|c|}{ Source of information } \\
\hline Design-brief & 1.34 & 5.55 \\
\hline Experimenter & 9.41 & 13.15 \\
\hline Self & 89.40 & 79.73 \\
\hline \multicolumn{3}{|l|}{ Operators } \\
\hline Add & 44.46 & 31.60 \\
\hline Evaluate & 13.61 & 11.10 \\
\hline Propose & 8.94 & 9.89 \\
\hline Comment & 3.32 & 11.10 \\
\hline Repeat & 1.58 & 1.33 \\
\hline Elaborate & 11.79 & 9.65 \\
\hline Justify & 2.85 & 2.29 \\
\hline Modify & 1.74 & 2.29 \\
\hline Qualify & 0.87 & 0.24 \\
\hline Read & 1.34 & 5.43 \\
\hline Request & 8.62 & 13.87 \\
\hline
\end{tabular}

Table 4. Aspects of design development considered by patient PF and normal control (NC) during different phases of design development

\begin{tabular}{|c|c|c|c|c|c|c|c|c|}
\hline & \multicolumn{2}{|c|}{$\begin{array}{c}\text { Problem } \\
\text { Structuring }\end{array}$} & \multicolumn{2}{|c|}{$\begin{array}{c}\text { Preliminary } \\
\text { Design }\end{array}$} & \multicolumn{2}{|c|}{ Refinement } & \multicolumn{2}{|c|}{ Detailing } \\
\hline & $P F$ & $N C$ & $P F$ & $N C$ & $P F$ & $N C$ & $P F$ & $N C$ \\
\hline People & $9 \%$ & $13 \%$ & $0 \%$ & $3 \%$ & $0 \%$ & $3 \%$ & $0 \%$ & $4 \%$ \\
\hline Purpose & $11 \%$ & $12 \%$ & $3 \%$ & $15 \%$ & $5 \%$ & $3 \%$ & $0 \%$ & $3 \%$ \\
\hline Behaviour & $25 \%$ & $17 \%$ & $0 \%$ & $12 \%$ & $2 \%$ & $3 \%$ & $0 \%$ & $5 \%$ \\
\hline Function & $13 \%$ & $11 \%$ & $25 \%$ & $21 \%$ & $22 \%$ & $34 \%$ & $0 \%$ & $7 \%$ \\
\hline Artefact & $42 \%$ & $47 \%$ & $72 \%$ & $49 \%$ & $71 \%$ & $58 \%$ & $0 \%$ & $81 \%$ \\
\hline
\end{tabular}


problem statement for information than is the control subject. The control self-generates more of the required information. The distribution of operator application is quite similar between patient and control. The only difference is that the patient exchanges many of the add operators for comment statements. The patient also utilises more request and read statements than the control. This is consistent with the source of information differences between patient and control ${ }^{5}$.

Lastly, at the end of the session, by way of an informal test of design-related memory, the experimenter asked the patient some questions about the lab space, for example, the colour of the carpet and walls, the number of shelves on the walls, the type of finish on the ceiling, etc. He answered all these questions correctly from memory. Furthermore, there were very few instances in the problemsolving session where the patient would introduce information into the problem space, and introduce it again at a later time (as measured by repeat statements in Table 4.

\section{DISCUSSION AND CONCLUSION}

The patient understood the task and even observed that "this is a very simple problem." His sophisticated architectural knowledge base was still intact and he used it quite skilfully during the problemstructuring phase. However, the patient's problemsolving behaviour differed from the control's behaviour in the following ways: (1) he was unable to make the transition from problem structuring to problem solving; (2) as a result, preliminary design did not start until two-thirds of the way into the session; (3) the preliminary design phase was minimal and erratic, consisting of three independently generated fragments; (4) there was no progression or lateral development of these fragments; (5) there was no carry-over of abstract information into the preliminary design or later phases; and (6) the patient did not make it to the detailing phase. In summary, he exhibited differential performance on different phases of the same task.

It is not possible to attribute patient and control differences to individual variations in problemsolving styles. The differences we observe here are consistent with patient and control differences reported in a recent group study involving 10 frontal lobe patients (Goel et al., 1997). In addition, there are many cognitive studies of experienced designers in similar problem-solving situations. Their performance (with respect to our measures) actually falls within a reasonably narrow band because of the common education and training they share (Akin, 1986; Cross, 1986; Eastman, 1969; Goel \& Pirolli, 1989, 1992; Goldschmidt, 1991). The performance of our control subject, as we have noted several times in the Results section, also falls within this band. PF's performance is simply very different from that of experienced architects. How is this difference to be explained?

There are three popular cognitive/computational explanations of frontal lobe dysfunction in the current literature: working memory (Goldman-Rakic, 1987), structured-event complexes (Grafman, 1989), and supervisory attentional system (Shallice, 1988). They are all based on classical von Neumann notions of compu-

\footnotetext{
${ }^{5}$ The novice control's session lasted 25 minutes, during which time he uttered 370 statements ( 15.4 statements/minute). Of these statements, $77 \%$ were devoted to design development. Within the design development phase the subject spent $48 \%$ of the statements on problem structuring and $52 \%$ on problem solving. Within the problem-solving phase, $46 \%$ of the statements were devoted to preliminary planning, $48 \%$ on refinement, and $6 \%$ on detailing. The subject had very limited drawing skills so he made paper cut-outs of desks and explored various configurations in an outline of the lab space. As is typical of novices, he engaged the task at a concrete, superficial level ... that of arranging furniture. There was no consideration of user activities, social concerns, circulation patterns, private and communal space, lighting, border conditions, etc. that preoccupied the trained architects. Yet in his arrangement of furniture, he engaged in two lateral transformations and one vertical transformation. The novice subject's short session, lengthened problem-structuring phase, failure to engage the problem at various levels of abstraction, and failure to pursue it through artefact detailing is simply a function of an impoverished knowledge base. The impoverished knowledge base does not prevent him from engaging in at least some lateral transformations. In contrast, the patient's knowledge base is intact (see main text and Table 4), so his difficulties require an alternative explanation.
} 
tation. Crudely, they claim that the mechanisms of working memory, long-term memory, or the central processing unit, respectively, are damaged by frontal lobe lesions. In evaluating the explanatory power of these theories for our data we must see (1) if there is evidence of damage to any of these mechanisms, and (2) if there is any evidence of differential involvement of these mechanisms in different phases of the task. The evidence for the former must come from patient data. Evidence for the latter must come from a theory of problem solving.

The working memory hypothesis (GoldmanRakic, 1987) claims that the patient's difficulty stems from his inability to hold information "on line" long enough for it to be processed and predicts differential performance in the task as working memory requirements vary. With respect to deficits in working memory, the patient did complain that his memory was not as good as it used to be, but his WMS-R scores are greater than 100, and he had ample access to memory aids throughout the session and utilised them. But even given working memory deficits it would also need to be the case that the preliminary planning phase of the task has greater working memory requirements than the problem-structuring phase. But in the absence of a theory of problem solving, there are no obvious reasons to believe this is the case, particularly when subjects have equal (and ample) access to external memory aids throughout the task.

The structured-event complex (SEC) theory (Grafman, 1989) proposes that much of our world knowledge is stored in script-like data structures and frontal lobe patients have difficulty in retrieving these structures. Its basic predication is that frontal lobe patients will have more difficulty with knowledge-rich tasks than knowledge-impoverished tasks. It further predicts that patients will have more difficulty retrieving low-frequency/lowexposure SECs compared to high-frequency/highexposure SECs, and that individual events (components) within an SEC can be retrieved because they are independentlystored (as single events) from the SECs.

Given the patient's performance during problem structuring, where he successfully drew upon a knowledge base every bit as rich and sophisticated as the expert control's, it is difficult to make a case for a general deficit in retrieving SECs. It is of course possible that there is selective impairment based upon frequency or exposure, but again, in the absence of theory, it is not obvious why there should be differential frequencies associated with different problem phases.

The supervisory attentional system (SAS) theory (Shallice, 1988) tries to account for patients' differential performance in routine and novel tasks by postulating dual control mechanisms. The idea is that there is a built-in contention scheduler that determines responses in over-learned, routine situations. However, when the organism is confronted with a novel situation, the contention scheduler is unable to cope and passes control to the more sophisticated supervisory attentional mechanism.

Any difficulties with top-down processing would emerge in our protocol as deviant control structure. But again, there was little evidence for a general deficiency in the patient's control structure - it was after all adequate to guide him during problem structuring - so again, the issue becomes one of how, in the absence of a theory, one can determine whether different problem-solving phases place differential demands on the SAS.

The challenge of explaining differential performance in phases of a single complex problem is a new one for these theories - and is indeed new to neuropsychology, given the short timescale of most diagnostic tests (Bechera et al., 1994; Goel et al., 1997; Shallice \& Burgess, 1991a, b). An interpretation of our results requires a theory of planning in ill-structured domains. We turn to Goel (1995) for such a theory. Goel articulates a fundamental difference in representational and computational structures involved in different phases of planning. But this distinction is quite radically different from those predicted by the above theories, at least on standard interpretations.

Goel (1995) characterises design problem solving as involving four development phases: problem structuring, preliminary design, refinement, and detail specification; and notes that each phase differs with respect to the type of information dealt with, the degree of commitment to generated ideas, the level of detail attended to, the number and types 
of transformations engaged in, and the symbol systems needed to support the different types of information and transformations.

What is of interest to us here is the contrast between the preliminary design phase and the refinement and detailing phases. Preliminary design is a classical case of creative, ill-structured problem solving. It is a phase where alternatives are generated and explored. This generation and exploration of alternatives is facilitated by the abstract nature of information being considered, a low degree of commitment to generated ideas, the coarseness of detail, and a large number of lateral transformations. A lateral transformation is one in which movement is from one idea to a slightly different idea rather than to a more detailed version of the same idea. Lateral transformations are necessary for the widening of the problem space and the exploration and development of kernel ideas (Goel, 1995). Our data suggest that the patient's ability to engage in such lateral transformations was compromised.

The refinement and detailing phases are more constrained and structured. They are phases where commitments are made to a particular solution and propagated through the problem space. They are characterised by the concrete nature of information being considered, a high degree of commitment to generated ideas, attention to detail, and a large number of vertical transformations. A vertical transformation is one in which movement is from one idea to a more detailed version of the same idea. It results in a deepening of the problem space (Goel, 1995).

Goel (1995) has argued that the ability to engage in lateral transformations is underwritten by a mechanism that supports ill-structured mental representations and computation. Informally, illstructured representations are imprecise, ambiguous, fluid, indeterminate, vague, etc. The ability to engage in vertical transformations is underwritten by a mechanism that supports well-structured mental representations and computation. Informally, well-structured representations are precise, distinct, determinate, and unambiguous. Furthermore, there is a computational dissociation between these two mechanisms (Goel, 1995). Our explanation for PF's performance (and the general dissocia- tion exhibited by frontal lobe patients in their lab and world performance) is that there is an anatomical dissociation corresponding to this computational dissociation. We postulate that PF's predominately right-hemisphere lesion has resulted in a selective impairment of the neural system that supports ill-structured representations and computations. This prevents him from successfully coping with the ill-structured preliminary design phase of our problem, and the ill-structured world at large. His computational and representational mechanisms for dealing with well-structured tasks (as measured by neuropsychological tests) remain intact.

Given our results and this explanation, we conclude that the right dorsolateral prefrontal cortex (DLPFC) (perhaps in conjunction with bilateral medial anterior cortex) is necessary for ill-structured representations and computations. This claim is consistent with both lesion and imaging data. At least five recent case studies (reviewed in Burgess, in press) have presented frontal lobe patients with an anatomical and neuropsychological profile similar to PF (Eslinger \& Damasio, 1985; Goldstein, Bernard, Fenwick, Burgess, \& McNeil, 1993; Shallice \& Burgess, 1991b). Like PF, these patients had difficulty in coping with real-world (ill-structured) situations despite high IQ. Three of these patients, like $\mathrm{PF}$, had lesions in the right DLPFC. In addition, several imaging studies (Elliot \& Dolan, 1998; Goel \& Dolan, 2000) also suggest that the right prefrontal cortex has a special role to play in openended (i.e., ill-structured) inference tasks with no right or wrong answers.

Manuscript received 4 January 1999 Revised manuscript received 9 July 1999 Revised manuscript accepted 19 October 1999

\section{REFERENCES}

Akin, O. (1986). Psychology of architectural design. London: Pion Ltd.

Bechera, A., Damasio, A.R., Damasio, H., \& Anderson, S.W. (1994). Insensitivity to future consequences following damage to human prefrontal cortex. Cognition, 50, 7-15. 
Burgess, P.W. (in press). Strategy application disorder: The role of the frontal lobes in human multitasking. Psychological Research.

Cross, N. (1986). Understanding design: The lessons of design methodology. Design Methods and Theories, 20(2), 409-438.

Damasio, H., \& Damasio, A.R. (1989). Lesion analysis in neuropsychology. Oxford: Oxford University Press.

Eastman, C.M. (1969). On the analysis of intuitive design processes. In G. Moore (Eds.), Emerging techniques in environmental design and planning. Cambridge, MA: MIT Press.

Elliott, R., \& Dolan, R.J. (1998). Activation of different anterior cingulate foci in association with hypothesis testing and response selection. Neuroimage, 8, 17-29.

Ericsson, K.A., \& Simon, H.A. (1984). Protocol analysis: Verbal reports as data. Cambridge, MA: MIT Press.

Ericsson, K.A., \& Simon, H.A. (1984). Protocol analysis: Verbal reports as data. (revised ed.). Cambridge, MA: MIT Press.

Eslinger, P.J., \& Damasio, A.R. (1985). Severe disturbance of higher cognition after frontal lobe ablation: Patient EVR. Neurology, 35, 1731-1741.

Goel, V. (1994). A comparison of design and nondesign problem spaces. Artificial Intelligence in Engineering, 9, 53-72.

Goel, V. (1995). Sketches of thought. Cambridge, MA: MIT Press.

Goel, V., \& Dolan, R.J. (2000). Anatomical segregation of component processes in an inductive inference task. Journal of Cognitive Neuroscience, 12, 1-10.

Goel, V., \& Grafman, J. (1995). Are frontal lobes implicated in "planning" functions: Interpreting data from the Tower of Hanoi. Neuropsychologia, 33(5), 623642.

Goel, V., Grafman, J., Tajik, J., Gana, S., \& Danto, D. (1997). A study of the performance of patients with frontal lobe lesions in a financial planning task. Brain, 120, 1805-1822.

Goel, V., \& Pirolli, P. (1989). Motivating the notion of generic design within information processing theory: The design problem space. AI Magazine, 10(1), 1836.

Goel, V., \& Pirolli, P. (1992). The structure of design problem spaces. Cognitive Science, 16(3), 395-429.

Goldman-Rakic, P.S. (1987). Circuitry of primate prefrontal cortex and regulation of behaviour by representational memory. In V.B. Mountcastle, F. Plum, \&S.R. Geiger (Eds.), Handbook of physiology: The nervous system Vol. 5 Higher functions of the brain, Part 1 (pp. 373-417). Washington, DC: American Physiological Society.

Goldschmidt, G. (1991). The dialectics of sketching. Creativity Research Journal, 4(2), 123-143.

Goldstein, L.H., Bernard, S., Fenwick, P., Burgess, P.W., \& McNeil, J.E. (1993). Unilateral frontal lobectomy can produce strategy application disorder. Journal of Neurology, Neurosurgery and Psychiatry, 56, 274-276.

Grafman, J. (1989). Plans, actions, and mental sets: Managerial knowledge units in the frontal lobes. In E. Perecman (Eds.), Integrating theory and practice in clinical neuropsychology Hillsdale, NJ: Lawrence Erlbaum Associates Inc.

Grafman, J. (1995). Similarities and distinctions among models of prefrontal cortical functions. In J. Grafman, K.J. Holyoak, \& F. Boller (Eds.), Structure and function of the human prefrontal cortex (pp. 337-368). New York: Annals of the New York Academy of Sciences.

Harlow, J.M. (1848). Passage of an iron rod through the head. Boston Medical Surgery Journal, 39, 389-393.

Harlow, J.M. (1863). Recovery after severe injury to the head. Publications of the Massachusetts Medical Society, 2, 327-346.

Heaton, R.K., Chelune, G.J., \& Talley, J.L. (1993). Wisconsin Card Sorting Test Manual. Odessa, FL: Psychological Assessment Resources.

Jensen, A.R., \& Rohwer, W.D. (1966). The Stroop Colour-Word test: A review. Acta Psychologica, 25, 36-93.

Kaplan, E.F., Goodglass, H., \& Weintraub, S. (1983). The Boston Naming Test (2nd ed.) Philadelphia, PA: Lea \& Febiger.

Lezak, M.D. (1995). Neuropsychological assessment (3rd ed.). New York: Oxford University Press.

Nelson, H.E. (1982). The National Adult Reading Test (NART): Test manual. Windsor, UK: NFER-Nelson.

Newell, A. (1990). Unified theories of cognition. Cambridge, MA: Harvard University Press.

Newell, A., \& Simon, H.A. (1972). Human problem solving. Englewood Cliffs, NJ: Prentice-Hall.

Penfield, W., \& Evans, J. (1935). The frontal lobe in man: A clinical study of maximum removals. Brain, 58, 115-133.

Reeves, D., Kane, R., \& Winter, K. (1994). Automated neuropsychological assessment metrics: Test administrator's guide. Washington, DC: Office of Military Performance Assessment Technology, Division of Neuropsychiatry, Walter Reed Army Institute of Research.

Reitman, W.R. (1964). Heuristic decision procedures, open constraints, and the structure of ill-defined 
problems. In M.W. Shelly \& G.L. Bryan (Eds.), Human judgements and optimality (pp. 282-315). New York: John Wiley \& Sons.

Samet, M.G., \& Marshall-Mies, J.C. (1987). Expanded Complex Cognitive Assessment Battery (CCAB): Final test administrator user guide (No. AAC-UM-33212). Alexandria, VA: Systems Research Laboratory, US Army Research Institute.

Schoenfeld, A.H. (1985). Mathematical problem solving. New York; Academic Press.

Shallice, T. (1982). Specific impairments of planning. Philosophical Transactions of the Royal Society of London, Series B, 298, 199-209.

Shallice, T. (1988). From neuropsychology to mental structure. Cambridge: Cambridge University Press.

Shallice, T., \& Burgess, P. (1991a). Higher-order cognitive impairments and frontal lobe lesions in man. In H.S. Levin, H.M. Eisenberg, \& A.L. Benton (Eds.), Frontal lobe function and dysfunction. Oxford: Oxford University Press.

Shallice, T., \& Burgess, P.W. (1991b). Deficits in strategy application following frontal lobe damage in man. Brain, 114, 727-741.

Spector, L., \& Grafman, J. (1994). Planning, neuropsychology, and artificial intelligence: Crossfertilization. In F. Boller \& G. Grafman (Eds.), The handbook of neuropsychology (Vol. 9) (pp. 377-392). Amsterdam: Elsevier.

Spreen, O., \& Strauss, E. (1991). A compendium of neuropsychological tests. New York: Oxford University Press.

Stuss, D.T., \& Benson, D.F. (1986). The frontal lobes. New York: Raven Press.

Wade, J.W. (1977). Architecture, problems and purposes: Architectural design as a basic problem-solving process. New York: John Wiley \& Sons.

Wechsler, D. (1981). WAIS-R manual. San Antonio, TX: The Psychological Corporation.

Wechsler, D. (1987). Wechsler Memory Scale-Revised manual. San Antonio, TX: The Psychological Corporation.

\section{APPENDIX A}

\section{Coding scheme}

Design Phase Level

The design phase level (see Figure 2) identifies the statement as either an experimental task (exp-task), self-monitoring (monitor), design development (design-development), or misc statement type, where each is defined as follows:

exp-task: Any statement having to do with the experimental design and setup. Example: Do you want me to read the problem statements out loud also?

monitor: Any statement used to take stock, further, review, or comment on the problem-solving process itself. Most of these correspond to what in the literature have been called metacognitive statement (Schoenfeld, 1985). Example: At this point I would like to explore the vertical space through a section.

design-development: Statements that advance the state of the design/artefact.

misc: Any statement that does not fall into one of the above categories.

The design development statements were further categorised into the following four subcategories.

problem-structuring: Statements that serve to solicit or generate information to structure the problem. Example: What is the one thing that bothers you the most about the existing layout? preliminary-design: Statements that result in the initial generation and exploration of some aspect of the design. They are accompanied by fast, fluid drawings. Example: Let's see what happens if we anchor the four corners with the principle scientists.

refine: Statements that serve to elaborate and further the commitment to an already-generated design idea or element. They are accompanied by transformations or variations of existing drawings. Example: This configuration results in undesirable through traffic past this workstation.

detail: Statements that serve to detail and give the final form to some aspect of the design. They are accompanied by crisp, straight-lined, dimensioned drawings using a hard pencil or pen. They often entail the use of precision drawing instruments, which were not available to our subjects. Example: The dimensions of the scientist's workstation are ...

These design development categories are quite common in the literature (Goel et al., 1997). They are relative to the time and resources available to the designer, but even with a session of short duration, it is possible to trace the development of the design and segment it into these phases.

Each design development category was further differentiated into the following aspects of design development subcategories: people, purposes, behaviour, function, and structure. As with the design development categories, these subcategories are also quite standard in the design literature. The ones employed here are adopted from Wade (1977). Briefly, the intuition behind them is that artefacts are designed to perform certain functions which are calculated to support certain behaviours, which help in the realisation of certain goals/purposes held by people/users. This categorisation provides an abstraction hierarchy linking users to artefacts and recognises that each intermittent step needs to be considered. 
people: Any statement dealing with the users of the artefact. Example: We need to accommodate 6 permanent and 10 temporary people.

purposes: Any statement dealing with the motives, intentions, and goals of the users. Example: Would you prefer a window position ...

behaviour: Any statement specifying the behaviour/activities the artefact is supposed to encourage and support. Example: Are meetings carried on in this space or elsewhere?

function: Any statement having to do with the desired, potential, or actual functionality of the artefact. Example: This shelf here will serve to disperse light ...

structure: Any statement having to do with the desired, potential, or actual form of the artefact. Example: The printer goes on top of this table along this wall.

misc: Any statement which does not fit into one of these categories.

\section{Episode Level}

Episodes are sequences of closely related statements united by a single problem-solving goal. Modules are episodes which have as their focus some aspect of the artefact/space being designed. Unlike the design phase level, the module level is problemspecific and thus dependent on particular tasks and subjects. The modules and submodules generated by our subjects appear in Table 3.

\section{Statement Level}

The third or statement level code has four independent fields; the operator applied, the content to which it is applied, the mode of the output, and the source of knowledge used. Operators are a labelling of statements by the function they serve in the problem space. While no theoretical commitment is made to any specific set, the 11 noted below are adequate for current purposes.

add: The basic operation of putting something into the problem space with some degree of commitment.

propose: Indicates that an idea is being entertained but is not yet committed to the problem space.

evaluate: Means that the statement is an explicit evaluation of a previous statement or design component in the problem space. comment: It is by and large the report of an activity rather than the execution of it. They generally occur with monitoring statements. Often it involves the subject explaining what he has just done, or just making some remarks which, while not directly related to his progress, are none the less illuminating.

modify: a statement which deletes or alters an existing idea or element that is already a part of the problem space. It is sometimes difficult to distinguish between add and modify, between an old idea being modified and a new idea being added. elaborate: Expands an existent idea or element.

justify: Offers a rationale for the addition, modification, or elaboration of ideas or elements in the problem space.

read: Any time the subject reads from the experimental task instructions, problem statement, or any other documents supplied with the task.

qualify: A statement used to hedge or further qualify the previous statement.

request: Statements used to ask questions of, or make suggestions to, the experimenter.

repeat: The application of the same operator to the same content again. Although any operator can be repeated, it is usually only add, modify, and elaborate questions that actually are repeated.

misc: Any statement that can't be coded with one of the above operators.

The content to which the operator is applied is also noted.

The mode of output of a statement is encoded as either verbal or written: Hand and facial gestures are not encoded.

verbal: Statements which are only uttered verbally, with no accompanying mark on paper.

written: Statements accompanied by marks on paper. These statements may or may not have an associated verbalisation.

Each statement was also encoded for the source of knowledge for the statement. The four categories used were the experimenter, the problem statement, self (retrieved from long-term memory), and inferred (deductively) from the information existent in the problem space.

experimenter: This is information that is either given to the subject by the experimenter, or actively solicited by the subject from the experimenter.

design-brief: This is information that the subject has obtained directly from the problem statement and any accompanying documents.

self: This is information that the subject either generates or retrieves from his long-term memory.

infer: This is information that the subject infers (in the strong deductive sense) from the information existing in the problem space. 